schiedliche mitochondriale Permeation schließen konnte.

Die von uns verwendeten Glycerinaldehyd-Konzentrationen führten fast immer zu einer erheblichen Beeinträchtigung sowohl der mitochondrialen Oxydation als auch der damit gekoppelten Phosphorylierung, so daß man selbst bei den unteren Konzentrationen von keiner echten Entkopplung der oxydati-

13 G. Bacigalupo, K. Rieche u. H. Wand, III. Intern. Congress of Chemotherapy, G. Thieme-Verlag, Stuttgart 1964, Vol. II, S. 1073. ven Phosphorylierung in vitro sprechen konnte. Eine Tendenz in Richtung einer echten Entkopplung zeigten die Ergebnisse der Hemmung der Glutamatoxydation von Tumormitochondrien. Die auffällige Störempfindlichkeit der Glutamatoxydation von Tumormitochondrien gegen entkoppelnde Agenzien haben wir schon früher beschrieben ${ }^{13,14}$.

${ }^{14}$ G. Bacigalupo u. H. Wand, Experientia [Basel] 20, 578 [1964].

\title{
Natur und Bildung des roten Farbstoffes in den Nierentubuli der Mutante "red" von Drosophila melanogaster*
}

\author{
Armin Wessing und Angelika Bonse \\ Physiologische Abteilung des Zoologischen Institutes der Universität Bonn \\ Herrn Professor Dr. Rolf Danneel zur Vollendung seines 65. Lebensjahres gewidmet
}

(Z. Naturforschg. 21 b, 1219—1223 [1966] ; eingegangen am 15. Mai 1966)

\begin{abstract}
Large quantities of 3-hydroxy-kynurenine (3-OHK) are accumulated in dilatations of the endoplasmic reticulum in the renal tubules of Drosophila melanogaster. In the tubules of the mutant "red", this stored $3-\mathrm{OHK}$ is converted into ommochromes, of which a small quantity is xanthommatin and a larger quantity is ommin; both of these stain the tubules red. The identification of these substances was confirmed by comparison with authentic compounds (figs. 1-3).

It has been found, by investigations with the electron microscope, that in larvae the stored 3-OHK is at first very compact. Later it becomes dispersed, and then is converted into ommochromes (figs. 5-9). After pupation, when the cells of the renal tubules become dehydrated and then undergo reconstruction, the ommochromes become condensed to the point of crystallization (figs. $10-14)$. During the conversion of the $3-\mathrm{OHK}$ in the cisterns of the endoplasmic reticulum, the surrounding membranes undergo a considerable growth, forming large vesicles (figs. 5-9). The ommochromes in their red, reduced form are stored in these vesicles. During the state of the pupa some of these vesicles are thrust out of the cells into the lumen (fig. 16).
\end{abstract}

Die Malpighischen Gefäße vieler landlebender Dipteren speichern fluoreszierende Stoffe. Am auffallendsten ist dabei das in granulärer Form akkumulierte gelbe 3 -Hydroxikynurenin ${ }^{1}$ sowie das Riboflavin. Bei vielen Mutanten sind Art und Gehalt an gefärbten Speicherstoffen verändert oder fehlen völlig ${ }^{2,3}$.

Im Jahre 1954 beschrieb Oster ${ }^{4}$ eine in seinen Zuchten spontan entstandene Drosophila-Mutante, die in den Nierentubuli einen roten Farbstoff bildet, und nannte sie "red" (red malpighian tubules 3-55,5士). Chromatographische Untersuchungen durch Aslaksen und Hadorn ${ }^{5}$ zeigten, daß bei dieser

\footnotetext{
* Die Untersuchung wurde mit dankenswerter finanzieller Unterstützung der Deutschen Forschungsge me in s ch af t durchgeführt.

1 A. Wessing u. R. Danneel, Z. Naturforschg. 16 b, 387 [1961].

2 K. S. Brehme u. M. Demerec, Growth 6, 351 [1942].
}

Mutante auch die Menge der Pterine in den Augen (Drosopterin, Sepiapterin, Isoxanthopterin) stark vermindert ist, die dadurch heller erscheinen. Durch weitere Untersuchungen, Kreuzungen und Transplantationen wiesen die Autoren nach, daß es sich bei dem in den Nierentubuli gespeicherten roten Farbstoff um ein Ommochrom handelt, das zwar nicht mit demjenigen der Augen identisch ist, aber wie dieses unter der Einwirkung der Gene $\mathrm{v}^{+}$und $\mathrm{cn}^{+}$gebildet wird.

Bei den vorliegenden Untersuchungen haben wir versucht, die Natur des roten Ommochroms zu ermitteln und seine Entstehung in den Nierentubuli

3 A. Wessing, Verh. Dtsch. Zool. Ges. Saarbrücken 1961; Zool. Anz., Suppl. 23, 166 [1962].

${ }^{4}$ I. I. Oster, DIS 28, 77 [1954].

5 E. Aslaksen u. E. Hadorn, Arch. Julius Klaus-Stiftg. Vererbungsforschg. 32, 464. [1957]. 
von Drosophila elektronenoptisch zu verfolgen, wobei besonderer Wert auf die Art der intrazellulären Farbstoffspeicherung gelegt wurde.

\section{Methoden}

Den roten Farbstoff gewannen wir aus den $\mathrm{Mal}$ p i g h i - Gefäßen älterer Puppen der Mutante „red“ * durch Präparation in R in g e r - Lösung und Aufquetschen der Tubuli von 250 oder 500 Tieren auf den Startpunkt des Chromatographiepapieres (Schleicher \& Schüll $2045 \mathrm{~b} \mathrm{M}$ ). Die Trennung erfolgte durch die für Ommochrome empfohlenen Gemische Collidin- $m / 2$ $\mathrm{KH}_{2} \mathrm{PO}_{4} 2: 3$ (obere Phase, aufsteigend) ${ }^{6,7}$ und Ameisensäure-Methanol-konz. Salzsäure ${ }^{8,9}$, verändert nach ${ }^{10}$ (80 [85-proz.]:15:0,5), ebenfalls aufsteigend. Diese Laufmittel erwiesen sich auch für unsere Versuche als besonders geeignet.

Nach der chromatographischen Auftrennung der Inhaltsstoffe wurden die Papierstreifen zunächst mehrere Stdn. mit fließendem Wasser, danach mit Butanol: Eisessig: Wasser (4:1:5) gewaschen. Bei Verwendung des Collidin-Gemisches wurden die Papiere vorher mit Aceton behandelt. Dadurch lassen sich alle anderen Stoffe außer den Ommochromen weitgehend entfernen.

Die Extraktion des roten Farbstoffes erfolgte zunächst mit 5-n. Salzsäure, danach mit konz. Ameisensäure. Um eine Veränderung des Ommochroms durch das Laufmittel nachzuprüfen, haben wir außerdem die Extraktion direkt aus dem Startfleck nach oben beschriebener Vorbehandlung vorgenommen.

Die Identifizierung der Ommochrome erfolgte durch Vergleich mit bekannten Substanzen ${ }^{11}$, deren Lichtabsorption in 5- $n$. HCl und Ameisensäure mit dem Zeiss-Spektralphotometer aufgenommen wurde.

Zur elektronenmikroskopischen Analyse fixierten wir die Gefäße 1 Stde. in $\mathrm{OsO}_{4}(1 \%)-\mathrm{K}_{2} \mathrm{Cr}_{2} \mathrm{O}_{7}(1 \%)^{12}+$ $0,6 \% \mathrm{NaCl}$ oder mit 6,25-proz. Glutaraldehyd-Na-Cacodylat-Puffer, $p_{\mathrm{H}} 7,1^{13}$ bei $4{ }^{\circ} \mathrm{C}$. Das erste Gemisch wurde dann 1 Stde. lang mit Drosophila-Ringer. Lösung, die zweite Fixierungslösung mehrere Stdn. mit Cacodylat-Puffer oder R inger-Lösung ausgewaschen und danach 1 Stde. mit 2-proz. $\mathrm{OsO}_{4}$ nachfixiert. Die Entwässerung erfolgte über mehrere Acetonstufen, wobei in 70-proz. Aceton $3 \mathrm{Stdn}$. lang mit einem Gemisch von 1-proz. Phosphorwolframsäure-1-proz. Uranylacetat nachkontrastiert wurde. Zur Einbettung verwendeten wir Vestopal W. Die Schnitte wurden mit einem LKB-Ultrotom II und die Aufnahmen am Siemens-ÜM 100d hergestellt ${ }^{14}$.

* Für die Überlassung der Mutanten danken wir dem Institute of Technology, Pasadena/California, USA.

6 A. Butenand, U. Schiedt, E. Biekert u. P. Kornmann, Liebigs Ann. Chem. 586, 217 [1954].

7 D. Bückmann, Z. Naturforschg. 18 b, 225 [1963].

$8+9$ A. Butenandt, E. Biekert u. B. Linzen, Hoppe-Seylers Z. physiol. Chem. 312, 227 [1958] ; 313, 251 [1958].

10 A. Kühn u. A. EgelhaAf, Z. Vererbungslehre 90, 244 [1959].

11 Für die Überlassung der verschiedenen Ommochrome danken wir Herrn Prof. Butenandt, Herrn Dr. Linzen und Herrn Dr. SchäFER vom Max-Planck-Institut für Biochemie,

\section{Ergebnisse}

\section{Versuche zur Identifizierung des roten Ommochroms}

Das „red"-Ommochrom läßt sich bei Chromatographie in Collidin- $\mathrm{KH}_{2} \mathrm{PO}_{4}$ nur z. T. als bräunlicher Fleck auf der Salzfront anreichern, der größte Teil verbleibt auf dem Start. Auf Grund der Ergebnisse von KüBLER ${ }^{15}$ und durch Vergleich mit Originalsubstanzen konnten wir Ommatin D und Rhodommatin ausschließen, nicht aber Xanthommatin und Ommin.

Der Vergleich eines HCl-Extraktes des Salzfrontfleckes mit gleichbehandeltem Xanthommatin ergab einander ähnliche Absorptionskurven (Abb. 1). Die

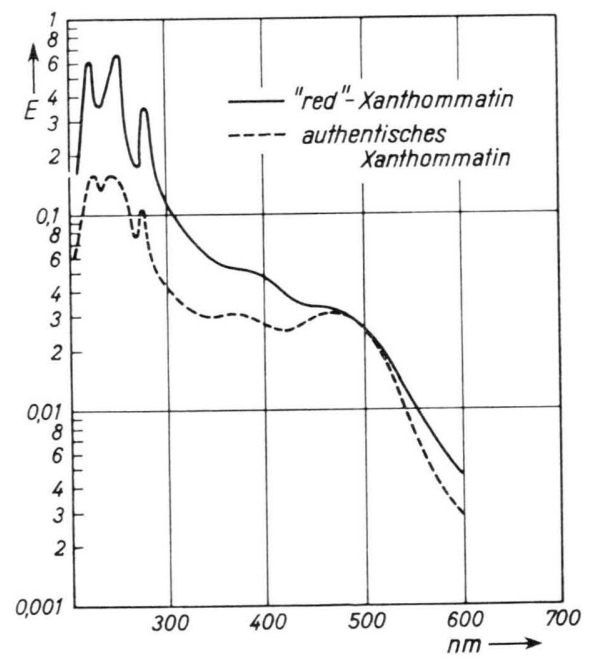

Abb. 1. UV-Spektren eines 5- $n$. HCl-Extraktes von authentischem Xanthommatin und Xanthommatin aus den $\mathrm{Mal}$. p i g hi schen Gefäßen der Mutante "red" nach Chromatographie mit Collidin- $m / 2-\mathrm{KH}_{2} \mathrm{PO}_{4}$.

geringfügigen Abweichungen der „red“-Kurve führen wir auf störende Stoffe zurück, die durch die vorhergehende Extraktion mit Wasser nicht entfernt worden waren.

Die weitere Analyse wurde mit Eluaten vorgenommen, die aus Chromatogrammen gewonnen und

München. Herrn Dr. Linzex haben wir auch für wertvolle Ratschläge bei der Durchführung der Untersuchungen zu danken.

12 K. E. Wohlfarth-Bottermann, Naturwissenschaften 44, 287 [1957].

13 D. D. Sabatini, K. Bensch u. R. J. Barrnett, J. Cell Biol. 17, 19 [1963].

14 Für die Benutzung des Mikroskopes danken wir Herrn Prof. Wohlfarth-Bottermans, Institut für Cytologie und Mikromorphologie der Universität Bonn. 
A. Wessing und A. Bonse, Natur und Bildung des roten Farbstoffes in den Nierentubuli der Mutante "red" von Drosophila melanogaster (S. 1219)
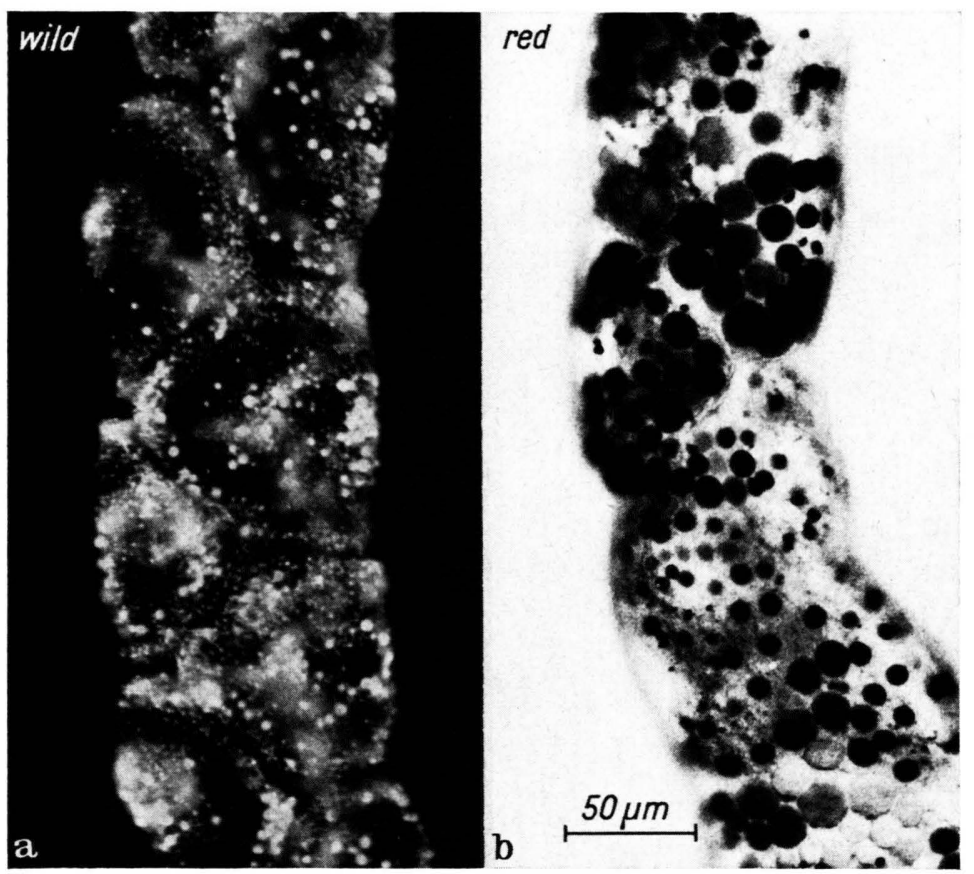

Abb. 4. Von den in den M a lpigh i schen Tubuli gespeicherten fluoreszierenden Substanzen leuchten bei fluoreszenzmikroskopischer Betrachtung der Wildform-Gefäße vor allem das 3-OH-Kynurenin und das Riboflavin auf (a), bei der Mutante „red“ sind dagegen die in großen Vesikeln liegenden roten Ommochrome auffallend, die das UV-Licht löschen
(Zeiss-Fluoreszenzmikroskop, UV-Licht $320-400 \mathrm{~nm}$, Erregerfilter Schott BG 38 und BG 3, bei Abb. 1 a Sperrfilter 53/47, Untergrund schwarz, bei Abb. 1 b Sperrfilter 44/47, Untergrund blau. Bei dieser Einstellung fluoreszieren 3-OHKynurenin und Riboflavin nicht!). 

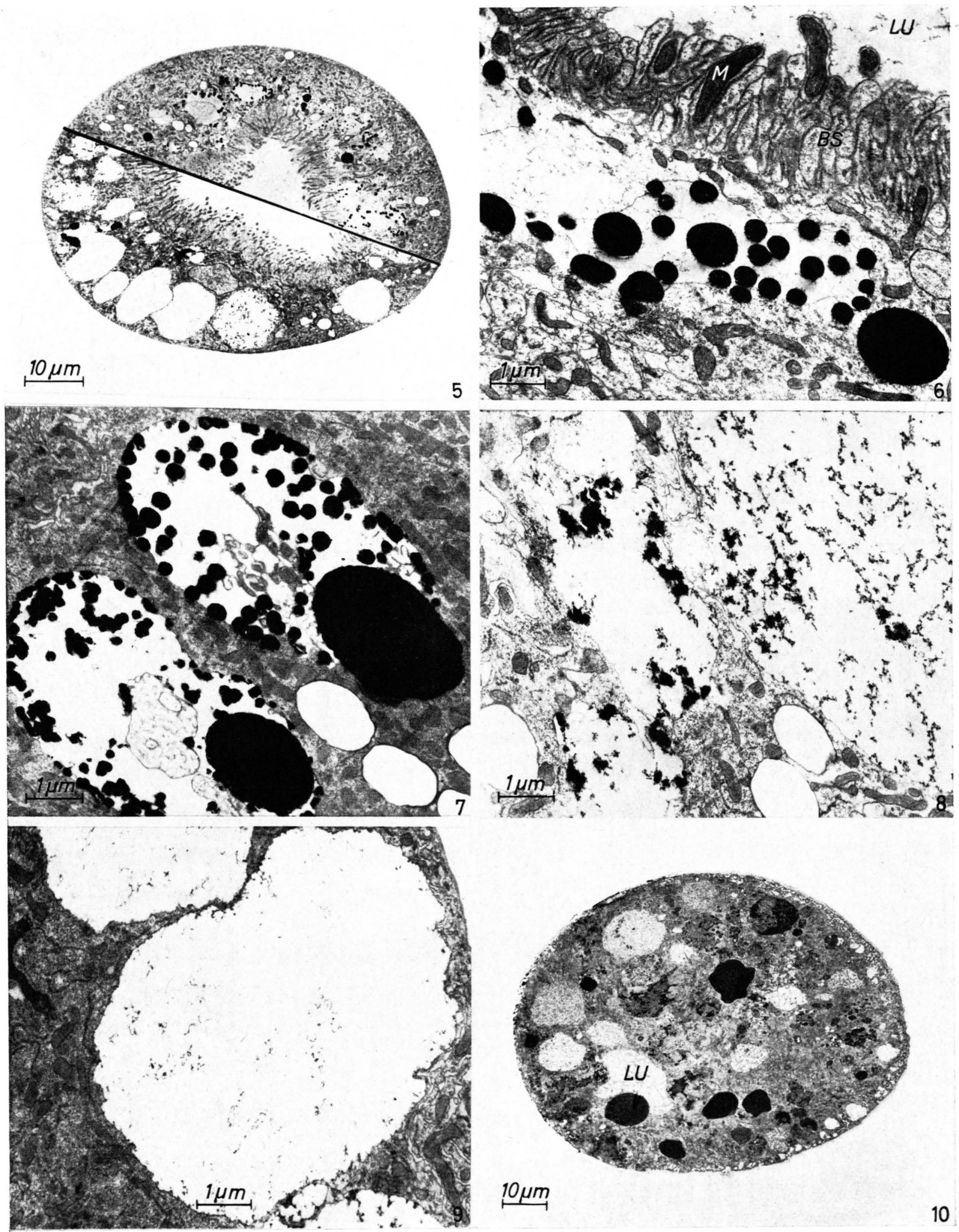

$10 \mu \mathrm{m}$ 

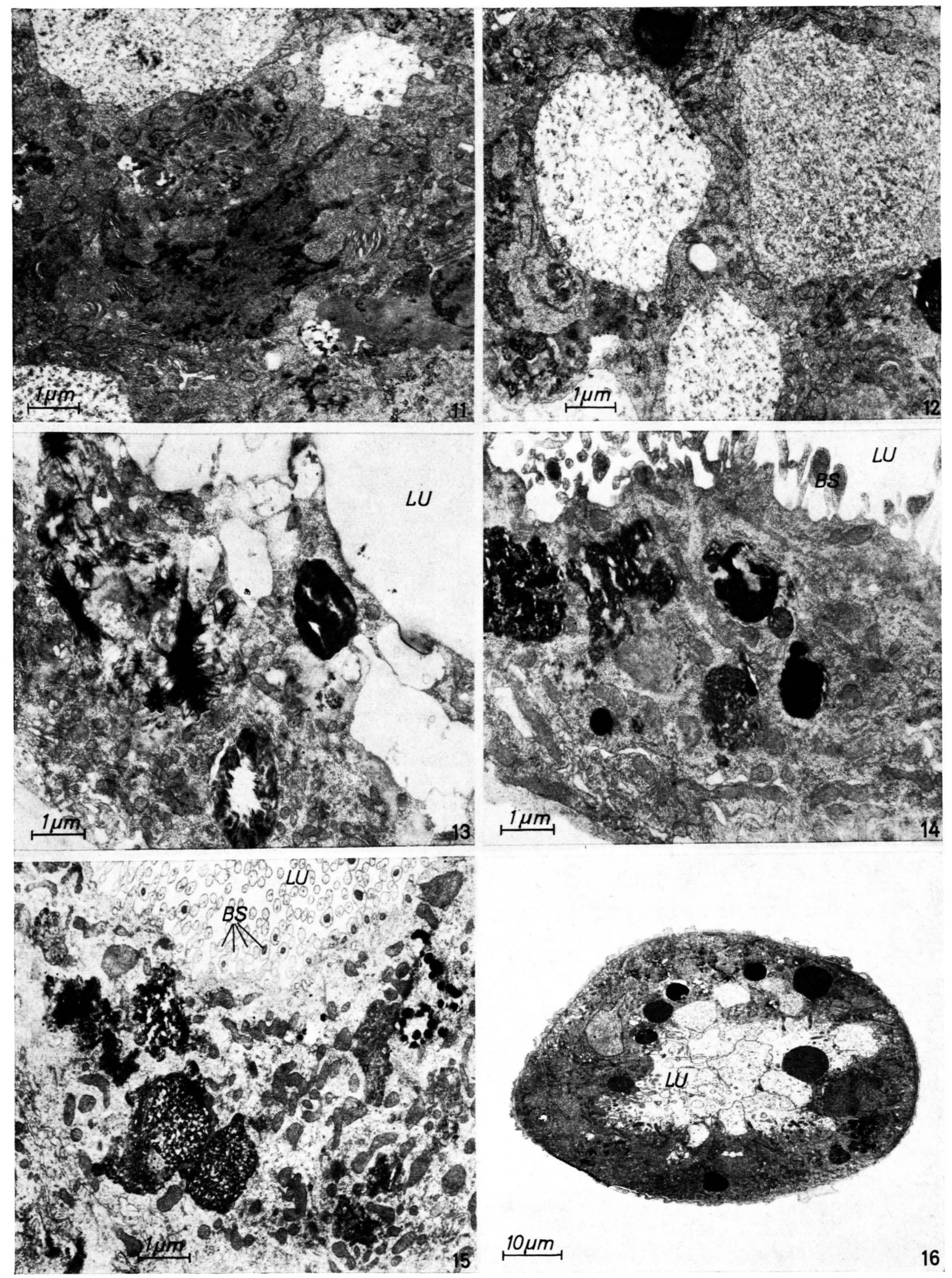

$10 \mu \mathrm{m}$ 
Abb. 5. Zwei halbe Querschnitte durch den proximalen Abschnitt eines larvalen Nierentubulus der Mutante „red" von Drosophila melanogaster, die die gespeicherten Pigmente in verschiedenen morphologischen Erscheinungsformen zeigen. Das rötliche Ommochrom und das 3-Hydroxikynurenin liegen in zahlreichen großen Vesikeln des intermediären Cytoplasmas in unterschiedlichen Dispersionsgraden. In der Mitte das von einem „Bürstensaum“ umgebene Gefäßlumen. (1000:1)

Abb. 6. Bei der Bildung des roten Ommochroms verteilt sich das in Erweiterungen des endoplasmatischen Retikulums liegende osmiophile 3-Hydroxikynurenin (rechts unten!) innerhalb großer Vesikel in kleinere Akkumulate $(\mathrm{LU}=\mathrm{Gefäß-}$ lumen, BS $=$ Zellzotten des Bürstensaumes, $\mathrm{M}=$ Mitochondrium).

Abb. 7. Zwei große Akkumulate von 3-Hydroxikynurenin (große schwarze Ballen!) zerfallen innerhalb zweier Vesikel in kleinere Einheiten.

Abb. 8. Die kleineren 3-Hydroxikynurenin-Akkumulate zerfallen allmählich. Dabei tritt offenbar die Umwandlung in Ommochrom ein.

Abb. 9. Große Vesikel mit feinverteiltem Ommochrom. (Abbn. $6-99500: 1$.

Abb. 10. Schrägschnitt durch den proximalen Abschnitt eines M a l p i g h i - Gefäßes der Puppe von ..red“. Durch die Veränderung des Zellaufbaues und die starke Entwässerung des Cytoplasmas wird das in den Vesikeln liegende Ommochrom konzentriert. ( $\mathrm{LU}=$ Lumen des Gefäßes; 880 : 1.)

Abbn. 11-14. Verschiedene Stufen der Konzentration des roten Ommochroms im Cytoplasma durch die starke Entwässerung der Zellen nach der Verpuppung. Der Inhalt der Vesikel wird immer dichter und kristallisiert allmählich in Nadeln aus $(\mathrm{LU}=$ Gefäßlumen, $\mathrm{L}=$ Leibeshöhle, $\mathrm{BS}=$ Mikrovilli des Bürstensaumes).

Abb. 15. In den Zellen der Imago liegen noch einige dichte Ansammlungen des Ommochroms aus der Larven- und Puppenzeit. Daneben wandelt sich das während der Imaginalzeit akkumulierte 3-Hydroxikynurenin wieder in Ommochrom um (rechts oben!). $(\mathrm{LU}=$ Lumen des Gefäßes, BS $=$ Querschnitte durch die Zellzotten des Bürstensaumes, $\mathrm{TM}=$ transformierte Mitochondrien. Abbn. $11-158500$ : 1.)

Abb. 16. Querschnitt durch den proximalen Abschnitt des Nierentubulus einer schlupfreifen Puppe. Die mit Ommochrom gefüllten Vesikel werden mit den umhüllenden Membranen in das Lumen (LU) abgegeben. (950 : 1.) 
mit Ameisensäure-Methanol-Salzsäure entwickelt worden waren. Synthetisches Xanthommatin liefert dabei mehrere Flecke, die allerdings nach Elution identische Absorptionskurven liefern. Authentisches Om. min A und Ommin aus Crangon- oder Bienenaugen lösten sich in diesem Laufmittel nur zögernd und zogen unter Schwanzbildung vom Startfleck mit blauvioletter Farbe bis etwa zum $R_{f}$-Wert 0,5. UV-Licht wird von beiden Substanzen gelöscht. Gleiches Verhalten zeigten bei derselben Behandlung auch die Ommochrome aus den Malpighischen Gefäßen von "red".

Durch Vorversuche mit den uns zur Verfügung stehenden reinen Substanzen konnten wir weiterhin feststellen, daß sich Ommin A nur zu einem verschwindend geringen Teil mit $5-n$. $\mathrm{HCl}$ aus dem Papier herauslösen läßt, während Xanthommatin dabei völlig in Lösung geht. Erst die Behandlung mit konz. Ameisensäure löst das Ommin vollständig aus dem Papier. Wir haben aus diesem Grunde die Chromatogramme von "red" nach der beschriebenen Vorbehandlung mit Wasser und mit alkoholischen Medien zunächst mehrere Stdn. lang mit 5-n. $\mathrm{HCl}$ zur Entfernung des Xanthommatins vorextrahiert. Die nachfolgende Behandlung mit konz. Ameisensäure löste dann den violetten Farbstoff leicht aus dem Papier. Ein Vergleich der Absorptionskurven mit derjenigen des authentischen Ommins A zeigte auch hier einen ähnlichen Verlauf (Abb. 2).

$\mathrm{Da}$ es sich beim „red"-Ommochrom vor allem um Ommin handelt, ließ sich auch durch ein verein-

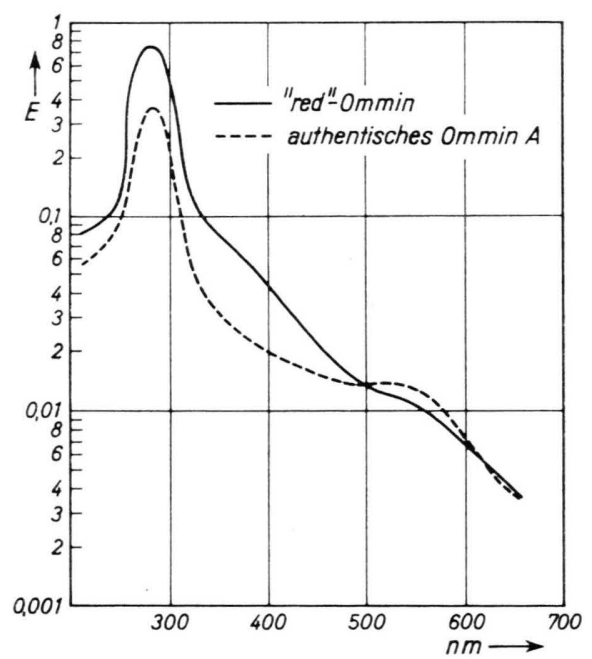

Abb. 2. Absorptionskurven von "red"-Ommin und authentischem Ommin A nach Chromatographie in $\mathrm{HCOOH}-\mathrm{CH}_{3} \mathrm{OH}-$ $\mathrm{HCl}$ und nachfolgender Elution in konz. $\mathrm{HCOOH}$. fachtes Verfahren nachweisen, wobei das Ommin mit Ameisensäure aus den auf das Papier aufgequetschten Tubuli nach gleicher Vorextraktion unmittelbar vorgenommen wurde (Abb. 3). Um ein meßbares

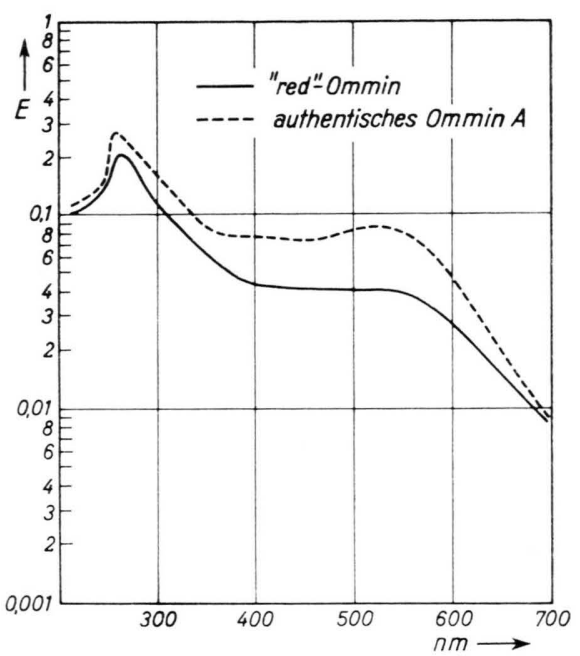

Abb. 3. Absorptionskurven von „red"-Ommin (1000 Puppen) und authentischem Ommin $\mathrm{A}$ in $\mathrm{HCOOH}$ ohne vorhergehende chromatographische Auftrennung.

Ergebnis zu erzielen, mußten für jeden Versuch die Malpigh i - Gefäße von mehreren tausend Puppen entnommen werden.

Die Unterschiede zwischen Ommin A und dem "red"-Ommin, die sich bei den Absorptionsmessungen zeigten, blieben auch nach dem Eindampfen der Eluate bestehen: Während das Ommin A seine dunkelviolette Farbe behielt, zeigte das "red“-Ommin eine hellere braun-violette Färbung.

Alle in den Nierentubuli von „red" auftretenden Ommochrome zeigen das für diese Stoffgruppe typische Verhalten ${ }^{16,6,7,8,9}$ : Bei der Behandlung mit Nitrit oder Wasserstoffsuperoxyd in essigsaurer Lösung färben sie sich durch Oxydation gelb und werden durch nachfolgende Behandlung mit Na-Dithionit oder Ascorbinsäure wieder zur roten Stufe reduziert. Die Reaktionen lassen sich sowohl durch Besprühen des Papiers als auch in Lösung durchführen.

\section{Elektronenoptische Analysen}

Schon die lichtmikroskopische Betrachtung läßt erkennen, daß das in den Zellen der larvalen Gefäße auftretende 3-Hydroxikynurenin (3-OHK) nur zu einem geringen Teil in die „red"-Ommochrome umgewandelt wird.

15 H. KüBLER, Untersuchungen zur Biogenese und Verbreitung der Ommochrome, Diss., Tübingen 1960. 
Das 3-OHK liegt normalerweise in Erweiterungen des endoplasmatischen Retikulums der intermediären Region der Zelle und bildet mehr oder weniger große Ansammlungen 1, 3, 17, 18, 19, 20 . Nach der Fixierung mit $\mathrm{OsO}_{4}$-haltigen Medien läßt sich das 3-OHK leicht durch seine Schwärzung identifizieren, die auf eine Reduktion des $\mathrm{OsO}_{4}$ zu metallischem Osmium bzw. zu niederen Oxydationsstufen zurückzuführen ist. Diese Reaktion kann man auch zur Identifizierung des 3-OHK auf dem Chromatogramm verwenden.

In den larvalen „red“-Zellen erweitern sich die Membranen, die die 3-OHK-Ansammlungen umgeben, zu mehr oder weniger großen Vesikeln (Abb. $\left.5^{*}\right)$, die gelegentlich die ganze basale und intermediäre Zellregion ausfüllen können.

Die Umwandlung des 3-OHK in die Ommochrome geht den elektronenmikroskopischen Bildern zufolge offensichtlich so vor sich, daß die runden Oxikynurenin-Akkumulate in immer kleinere runde Ansammlungen zerfallen (Abbn. 6, 7) und schließlich in eine feindispersere, amorphe Erscheinungsform übergehen (Abbn. 8, 9). Bei dem letzten Schritt erfolgt die Umwandlung in die Ommochrome. Dies ergibt sich aus dem Verhalten bei der Glutaraldehyd-Fixierung, wobei zwar das 3-OHK, nicht aber die Ommochrome aus der Zelle gelöst werden: Die meisten kleinen und mittleren Vesikel erscheinen danach bis auf geringfügige Niederschläge leer und nur in den großen Vakuolen verbleibt der amorphe feine Niederschlag. Die Umwandlung des 3-OHK in die Ommochrome geht demnach mit der Vergrößerung der Vesikel, die das Chromogen speichern, parallel.

Ein von den normalen Verhältnissen stark abweichendes Bild bieten die Zellen der Puppengefäße. Bei der Wildform verschwindet nach der Verpuppung das gespeicherte $3-\mathrm{OHK}$ bis auf geringe Reste aus den Gefäßen und dient vermutlich zum Aufbau des Xanthommatins in den Augen der wachsenden Imago. In den Zellen der Nierentubuli erscheinen dann leere Vakuolen, die allmählich eingeschmolzen werden. Die Tubuluszellen erfahren außerdem nach

16 E. Becker, Z. ind. Abst. Vererbungslehre 80, 157 [1942].

17 A. Wessing, Protoplasma 55, 264 [1962].

18 A. W Essing, Protoplasma 56, 433 [1963].

19 A. Wessing, in: Funktionelle und morphologische Organisation der Zelle, Sekretion und Exkretion. 2. wiss. Konf. Ges. Naturf. u. Ärzte, Reinhardsbrunn 1964, Berlin, Heidelberg, New York 1965, S. 228.

20 A. Wessing, Naturwiss. Rdsch. 19, 139 [1966].

* Abbn. 4-16 s. Tafel S. 1220 a-c. der Verpuppung einen tiefgreifenden Umbau ihrer Zellarchitektur, bei der das Cytoplasma mit allen Organellen immer stärker kondensiert wird ${ }^{18}$.

Von dieser Entwässerung und Veränderung des Cytoplasmas werden auch die Puppengefäße von "red" erfaßt, wobei die feinverteilten Ommochrome in den sich verkleinernden Vesikeln immer dichter werden (Abb. 10-12), bis schließlich die Ommochrome bei der weiteren Kondensation allmählich in kurzen Nadeln auskristallisieren (Abbn. 13, 14).

Die Umwandlung des 3-OHK in die „red"-Ommochrome ist, wie schon Aslaksen und Hadorn beschrieben haben, kurz nach der Verpuppung am stärksten. Durch Fixierung mit Glutaraldehyd kann man darüber hinaus nachweisen, daß der überwiegende Teil des eingelagerten elektronendichten Pigmentes aus Ommochromen besteht. Daß Ommochrome bei dichter Packung sehr elektronendicht erscheinen, lehren die Befunde über den Feinbau der Augen von Dipteren und anderen Insekten 21-26 u. a. Ommatine und Ommine verhalten sich in dieser Hinsicht gleich.

Ein Teil dieses Materials wird später in das Gefäßlumen abgegeben. Dabei wandern die Vesikel lumenwärts und lösen sich aus dem Cytoplasma heraus, bleiben aber von einem schmalen Cytoplasmasaum umhüllt (Abb. 16). Sie wandern dann mit den Exkreten zusammen in den Enddarm, den sie leicht rötlich färben. Innerhalb des Gefäßlumens können sich die Vesikel öffnen und ihren Inhalt in das Lumen ergießen.

Ein großer Teil der Ommochrom-Ansammlungen überdauert jedoch die Puppenzeit in den intrazellulären Vesikeln. Diese Akkumulate kann man daher in den Zellen der Imagines neben neuen in Umwandlung begriffenen 3-OHK-Ansammlungen wiederfinden (Abb. 15).

\section{Diskussion}

Die Malpighi-Gefäße vieler landlebender Dipteren erfüllen neben ihrer exkretorischen Tätigkeit noch eine andere Funktion: Sie speichern nämlich in den Zellen der reabsorbierenden Gefäß-

21 R. Danneel u. B. Zeutzschel, Z. Naturforschg. 12 b, 580 [1957].

22 D. J. Nolte, Heredity 16, 25 [1961].

23 D. J. Nolte, South African J. Sci. 57, 121 [1961].

24 E. EGuchi, J. Ultrastruct. Res. 7, 328 [1962].

25 L. Schneider u. H. Langer, Z. Naturforschg. 21 b, 196 [1966].

${ }^{26}$ H. Fuge, Naturwissenschaften 53, 136 [1966]. 
abschnitte eine große Anzahl von wichtigen Stoffen, wie z. B. Fette, Aminosäuren und viele fluoreszierende Substanzen. Der größte Teil dieser Stoffe verschwindet nach der Verpuppung aus den Zellen und wird wahrscheinlich beim Aufbau der Imago verwendet. Eine dieser Substanzen ist das 3-Hydroxikynurenin, das Chromogen der braunen Augenpigmente, der Ommochrome.

Bei den cycloraphen Dipteren * tritt normalerweise die Umwandlung des 3-OHK in das Ommochrom nur in den Pigmentzellen der Ommatidien ein. Die hier beschriebene Drosophila-Mutante „red“ macht davon eine Ausnahme, da bei ihr schon das in den Nierentubuli eingelagerte $3-\mathrm{OHK}$ in Ommochrom verwandelt wird, ein Vorgang, der bei den Lepidopteren die Regel zu sein scheint, bei denen das Ommochrom, wie bei der Drosophila-Mutante „red“, vorwiegend in seiner roten, reduzierten Form in den Zellen der Nierentubuli auftritt und während der Puppenzeit als Exkret ausgeschieden wird ${ }^{27,28}$.

Ursprung, Graf und Anders ${ }^{29}$ und Hertweck ${ }^{30}$ wiesen nach, daß nach einer unspezifischen Schädigung der Malpighi-Gefäße auch in den Zellen von Wildtieren Ommochrom entstehen kann. Diese Befunde stimmen mit unseren Erfahrungen überein: Gelegentlich sind nämlich die Tubuli von schlecht ernährten Wildtieren aus alten Kulturen rot gefärbt. Wie elektronenmikroskopische Bilder von Wild-Gefäßen zeigen, geht aber auch bei normal ernährten Tieren das akkumulierte 3-OHK an einigen Stellen in einen Stoff über, der im Elektronenmikroskop wie „red"-Ommochrom aussieht. Auch der Fettkörper von Drosophila, der ja Kynurenin speichert $31-33$ u. a. kann nach einer Schädigung rotes Ommochrom bilden ${ }^{30}$. Bei der Drosophila-Mutante „red cells" bilden die Fettzellen ebenfalls rote $\mathrm{Om}$ mochrompigmente ${ }^{34}$. Eine andere von CLANCY ${ }^{35}$ beschriebene Mutante (In(2LR)40d) reichert in den Nierentubuli rotes Pigment an, das nach Meinung des Autors aus den degenerierenden Augen stammt.

Offensichtlich genügt also schon ein kleiner stoffwechselphysiologischer Anstoß, um das in den Malpighi-Gefäßen und im Fettkörper gespei-

* Becker ${ }^{16}$ erwähnt, daß sich bei der (orthoraphen) Mücke Phryne in den M a l p i g h i - Gefäßen Ommatin befindet.

27 R. Wolfram, Z. Naturforschg. 3 b, 291 [1948].

28 R. Wolfram, Z. Vererbungslehre 83, 254 [1949].

29 H. Ursprung, G. E. Graf u. G. Anders, Rev. Suisse Zool. 65, 449 [1958].

30 H. Hertweck, Folia biol. [Praha] 6, 293 [1960].

31 T. M. Rızкі, J. biophysic. biochem. Cytol. 9, 567 [1961].

32 T. M. Rizki u. R. M. Rizki, J. Cell Biol. 17, 87 [1963]. cherte Kynurenin bzw. Hydroxikynurenin in Ommochrom zu verwandeln, ein Syntheseschritt, der auch in vitro gelingt (Zusammenfassung bei l.c. ${ }^{36}$ ). Nach den Untersuchungen der Butenandtschen Schule ${ }^{36,15}$ kommt bei den Dipteren nur das im oxidierten Zustand gelbbraune Xanthommatin vor und zwar nur in den Augen. Die Mutante "red" von Drosophila bildet also eine Ausnahme, da sich bei ihr ein rotviolettes, schwerlösliches Ommin nachweisen ließ. Die beobachteten, geringfügigen Unterschiede zwischen diesem Ommin und dem zum Vergleich benutzten Ommin A sind wohl darauf zurückzuführen, daß die Ommine nach den bisherigen präparativen Erfahrungen ${ }^{37}$ nicht einheitlich sind und wahrscheinlich von Tierart zu Tierart variieren.

Ommin konnte bisher noch nicht kristallin gewonnen werden ${ }^{8}$. In den Zellen der entwässerten „red"-Puppengefäße kristallisiert es jedoch zu Büscheln verfilzter Nadeln, verhält sich in dieser Hinsicht also wie das Rhodommatin ${ }^{38}$.

Die Umwandlung des in Erweiterungen des endoplasmatischen Retikulums gespeicherten 3-OH-Kynurenins in Ommin und Xanthommatin vollzieht sich in den Zisternen des Retikulums. Dabei blähen sich die Chromogen umhüllenden Membranen immer weiter auf. Dieses intensive Membranwachstum wird offensichtlich durch chemische Umwandlung des im Retikulum akkumulierten Stoffes in seine Folgeprodukte veranlaßt, hier durch Kondensation des 3-OHK in die Ommochrome.

Die Abgabe des Ommochroms in das Gefäßlumen durch die Puppenzelle, die nach Art einer merokrinen Sekretion erfolgt, läßt erkennen, daß die Zelle bestrebt ist, die bei „red" bestehende, genbedingte Stoffwechselanomalie, die nach der elektronenmikroskopischen Analyse zu einer Zellschädigung führt, zu kompensieren. Eine solche Ausscheidung von unerwünschten Speicherprodukten aus den Nierenzellen in das Lumen ist für Drosophila durchaus ungewöhnlich und kommt nach unseren Beobachtungen nur unter anomalen Verhältnissen vor, nämlich z. B. dann, wenn man Fremdstoffe in die Hämolymphe injiziert.

33 T. M. Rizki u. R. M. Rizki, J. Cell Biol. 21, 27 [1964].

34 J. C. Jones u. E. B. Lewis, Biol. Bull. 112, 220 [1957].

35 C. W. Clancy, Genetics 40, 567 [1955].

36 A. Butenandt, E. Biekert, H. Kübler u. B. Linzen, HoppeSeylers' Z. physiol. Chem. 319, 238 [1960].

37 A. Butenandt, G. Neubert u. U. Baumann, Hoppe-Seylers' Z. physiol. Chem. 314, 15 [1959].

38 A. Butenandt, E. Biekert, H. Kübler, B. Linzen u. P. Traub, Hoppe Seylers' Z. physiol. Chem. 334, 71 [1963]. 